\title{
Heat and Mass Transfer in Fires: Scaling Laws, Ignition of Solid Fuels and Application to Forest Fires
}

\author{
Jose L. Torero ${ }^{1}$ and Albert Simeoni ${ }^{*}, 2$ \\ ${ }^{I}$ BRE Centre for Fire Safety Engineering, The University of Edinburgh, Edinburgh, United Kingdom \\ ${ }^{2}$ CNRS UMR 6134 - Sciences Pour l'Environnement, University of Corsica, Corte, France
}

\begin{abstract}
Fire is a phenomenon that covers a multiplicity of scales depending on the different processes involved. Length scales range from the nanometres when addressing material flammability to the kilometres when dealing with forest fires, while time scales cover a broad spectrum too. Heating of structural elements can be measured in hours while characteristic chemical times for reactions do not exceed the millisecond. Despite these wide ranges, a series of simple scaling laws seem to describe well a multiplicity of processes associated with fire. In this paper, flaming ignition of a solid fuel will be presented within the context of general scaling laws and forest fires. Therefore, the case of highly porous vegetable fuels will be investigated to extend the theory to the forest fires application.
\end{abstract}

Keywords: Wildland fires, scaling, ignition.

\section{INTRODUCTION}

Scaling analysis for fires has been the subject of numerous papers and reviews. Many subjects have been addressed in great detail providing a series of scaling laws that are currently used for many scientific and engineering applications. Attempts to develop comprehensive sets of non-dimensional parameters have relied on common techniques such as the Buckingham Pi theorem to define a series of non-dimensional parameters. A classic paper that follows this method is that by Quintiere [1].

Despite the rigorous attempts towards establishing a comprehensive list of non-dimensional parameters, the development of scaling parameters for fire has followed a different path. Scaling and non-dimensional parameters have been mostly derived based on the particular application. This has been the case mostly because the different problems associated to fire cover an extremely wide variety of length and time scales. Thus, a single set of scaling parameters seems to be impossible.

The most classic attempts at scaling are associated with pool fires, entrainment and compartment fires. These are reviewed to a great depth by Zukoski [2]. The main parameter extracted from Zukoski's analysis is

$Q^{*}=\frac{\dot{Q}_{0}}{\rho_{\infty} T_{\infty} C p_{\infty}(g D)^{1 / 2} D^{2}}$

Where $\dot{Q}_{0}$ is the energy release rate issued from the combustion process, $\rho_{\infty}$ the ambient density, $T_{\infty}$ the ambient temperature, $C p_{\infty}$ the ambient specific heat, $\mathrm{g}$ the gravity vector

\footnotetext{
*Address correspondence to this author at the CNRS UMR 6134 - Sciences Pour l'Environnement, University of Corsica, Corte, France;

Tel: +33 495450 638; Fax: +33 495450 162; E-mail: simeoni@univ-corse.fr
}

and $\mathrm{D}$ the diameter or characteristic length scale. Q* represents the ratio between the energy provided by the combustion reaction and the energy associated with the induced buoyant flow. It hides within the parameter a number of characteristic values like the buoyantly induced velocity:

$u_{b}=\sqrt{g D}$

And the assumption that the pool diameter is the characteristic length scale of the problem. This choice of length scale serves to quantify the large scale motion within a fire but is not a representative scale for the combustion region, radiation or for turbulence. Thus it can not allow scaling phenomena such as flickering or radiative heat transfer. A set of different length scales is presented by Joulain in his review [3].

A different approach to $\mathrm{Q}^{*}$ is to make it of order unity and use it to extract a characteristic length scale

$$
L=\left(\frac{\dot{Q}_{0}}{\rho_{\infty} T \phi_{\infty} C p_{\infty} \sqrt{g}}\right)^{2 / 5}
$$

This length scale " $L$ " can then be successfully used to scale the flame height and the temperature distribution at the axis of a pool fire. This does not require the definition of the length scale, but it implies that motion is purely dominated by buoyancy. This has been found to be limited when either fuel injection velocity (jets), geometry (confinement) or length scale (flow instabilities) introduced other driving forces to the problem. Delichatsios conducts a detailed extension to the above method in terms of what he labels as the Fire Froude number [4]. Where the Froude number does not follow the classical definition, $\mathrm{Fr}=\mathrm{u} /(\mathrm{gD})^{1 / 2}$ but a more complex definition linked to the energy release rate. Porous fuels represent a particular limitation to this approach be- 
cause the characteristic length scale is affected by the nature of the fuel.

The work on pool fires, entrainment and its effects on compartment fires has been explored in great detail by many reviews and is the subject of numerous pages in text books [5] and handbooks [4], thus will not be the subject of this paper. Nevertheless this analysis serves well as an introduction because it explains well many of the issues related to the scaling of fires. Simple relationships tend to describe well some basic phenomena, nevertheless as the analysis becomes more detailed, these simple expressions begin to break down and different parameters become relevant. In this paper a problem where the use of scaling is less common will be addressed: material ignition. The first section will present material flammability. In Section 2, a general model for flaming ignition of solid fuel will be presented and applied to different types of fuels. Scaling of experimental results, obtained at laboratory scale will be also discussed. Then, the extension of the model to forest fuels will be presented and the validity of the model will be discussed.

\section{MATERIAL FLAMMABILITY}

Proper evaluation of material flammability requires understanding of the flame structure, the degradation process of the material and the interface (boundary condition) between the two. A simple model for the ignition process based on previous studies will be used here [6-13]. The following description and the equations developed in the next section can be found in [12].

When the material, initially at $\mathrm{T}_{\infty}$, is subject to a heat insult $\left(\dot{q}_{e}^{\prime \prime}\right)$ the temperature rises and a temperature distribution function of the location and time is created inside the material $(T(x, t))$. The surface temperature $\left(T_{S}(0, t)\right)$ will increase but the material will not release any flammable gases (Fig. 1(a)) until a pyrolysis temperature is attained $\left(\mathrm{T}_{\mathrm{P}}\right)$ (Fig. 1(b)). The time necessary to achieve the pyrolysis temperature is generally referred as the time to pyrolysis, $t_{p}$. Throughout the pre-heating period the fuel concentration in the gas phase can be considered negligible. The absence of gas phase fuel does not preclude degradation of the material, generally, throughout the preheating process, the material degrades and subsequently its thermal properties change. Once the pyrolysis temperature is attained the fuel concentration increases until it attains a "lean flammability limit" $\left(\mathrm{Y}_{\mathrm{F}, \mathrm{L}}\right)$. The time necessary to reach this fuel concentration is called the "mixing time" or "time to attain a flammable mix-

(a)

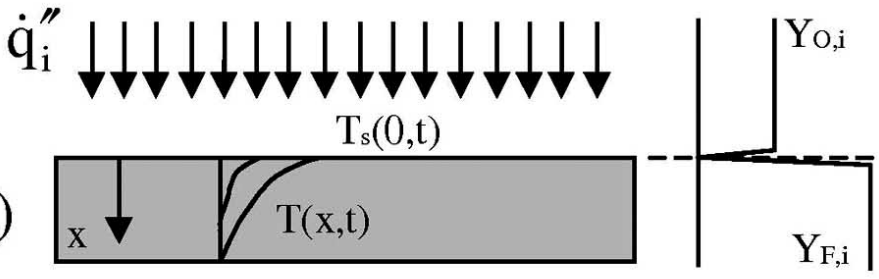

(b)

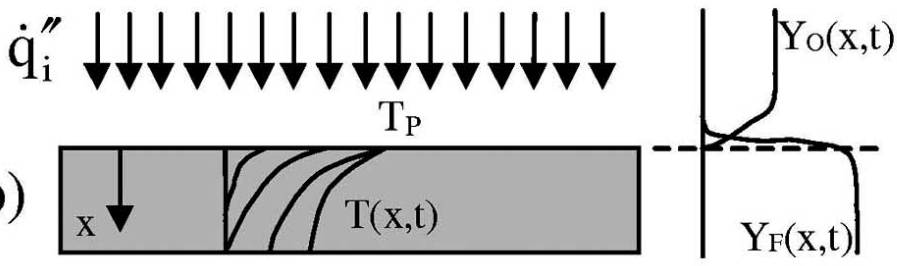

(c)
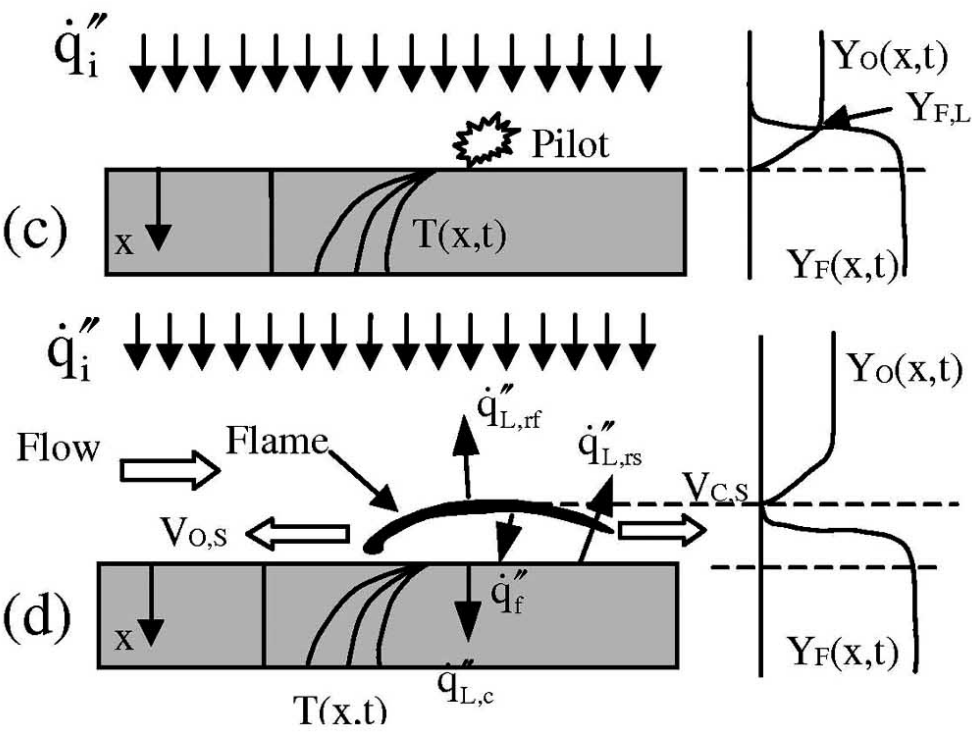

Fig. (1). Schematic of the sequence of events leading to ignition and growth of a fire over a combustible surface. $Y_{O}, Y_{F}$ and $Y_{F, L}$ being the mass fractions of oxygen, fuel and fuel corresponding to the flammability limit, respectively. The subscript $\mathrm{i}$ refers to initial conditions. 
ture" $\left(t_{m}\right)$. At this point, the temperature of the gases rises until a self-sustained exothermic reaction is attained. This period is called the "induction time" $\left(\mathrm{t}_{\mathrm{i}}\right)$ and can be achieved by heating of the mixture (auto-ignition) or by means of a pilot or hot spot (piloted ignition). Piloted ignition is illustrated in Fig. 1(c).

It is important to note that after pyrolisis is initiated the net heat flux to the surface is used entirely for pyrolisis and no subsequent temperature increase is noted. At this point a flame might not establish over the surface of the fuel because the pyrolysis rate remains too small to sustain a flame, this period is characterized by flashing. The pyrolysis rate will increase with time increasing the frequency of the flashing until a flame is fully established. Once a flame is established the growth process follows. In the presence of a flow (i.e. HVAC induced flows) spread can be of two types, opposed $\left(\mathrm{V}_{\mathrm{O}, \mathrm{S}}\right)$ and co-current $\left(\mathrm{V}_{\mathrm{C}, \mathrm{S}}\right)$. Opposed flame spread goes against the flow and co-current in the direction of the flow (Fig. 1(d)). The flame enhances the heat feedback to the unburned surface increasing its temperature to $T_{p}$, leading to the production of flammable gases and resulting in subsequent pilot ignitions. For spread, the existing flame can be considered the pilot. Opposed and co-current spread are complex phenomena, the former related to leading edge characteristics and the latter depending on the flame geometry and characteristics. The net heat supply to the surface $\left(\dot{q}_{f}^{\prime \prime}\right)$ is established by the flow structure, the heat generated by the flame $\left(\dot{q}_{g}^{\prime \prime}\right)$ and radiative losses $\left(\dot{q}_{L, f f}^{\prime \prime}\right)$. A fraction of this heat is used for fuel pyrolysis $\left(\dot{q}_{S}^{\prime \prime}\right)$ and the rest is lost to the flow, by radiation from the surface to the environment $\left(\dot{q}_{L, r s}^{\prime \prime}\right)$ or through the material by conduction $\left(\dot{q}_{L, c}^{\prime \prime}\right)$. Although heat supply is controlled by gas phase dynamics, the preheating process is controlled by the thermal properties of the degrading material.

\section{IGNITION}

Based on the above model, and approximate evaluation of the ignition delay time $\left(\mathrm{t}_{\mathrm{ig}}\right)$ can be done by independent evaluation of all three characteristic times and their subsequent addition

$$
t_{i g}=t_{p}+t_{m}+t_{i}
$$

Under fast chemical kinetic conditions (low gas velocities and elevated oxygen concentrations), introducing a strong pilot reduces the induction time $\left(\mathrm{t}_{\mathrm{i}}\right)$ making it negligible when compared to $t_{p}$ and $t_{m}$. Also, the period where the transient evolution of the fuel concentration in the gas phase increases towards a flammable mixture $\left(\mathrm{t}_{\mathrm{m}}\right)$ has been commonly considered short when compared to heating of the solid fuel sample. Therefore, the fuel and oxidizer mixture has been normally considered to become flammable almost immediately after pyrolysis starts. Fig. (2) provides data obtained using black PMMA as fuel that, although shows some discrepancy, especially for $\dot{q}_{e}^{\prime \prime}<20 \mathrm{~kW} / \mathrm{m}^{2}$, serves to justify this assumption. Pyrolysis temperatures and times are thus commonly referred to as ignition temperature $\left(\mathrm{T}_{\mathrm{ig}}\right)$ and ignition delay time $\left(\mathrm{t}_{\mathrm{ig}}\right)$ respectively [6,7], and equation (4) simplifies to $t_{i g}=t_{p}$, and $T_{i g}$ can be defined as $T_{p}$.

Under these assumptions the solid heating process is described by the energy equation:

$$
\begin{array}{ll}
\frac{\partial^{2} T}{\partial x^{2}}=\frac{1}{\alpha} \frac{\partial T}{\partial t} & \begin{array}{l}
\mathrm{x}=0,-k \frac{\partial T}{\partial x}=\dot{q}_{S}^{\prime \prime}(0, t) \\
t=0 \\
x \rightarrow \infty
\end{array} \quad T=T_{\infty}
\end{array}
$$

The boundary conditions assume that the heat available for pyrolysis (also called net heat flux) at the surface is

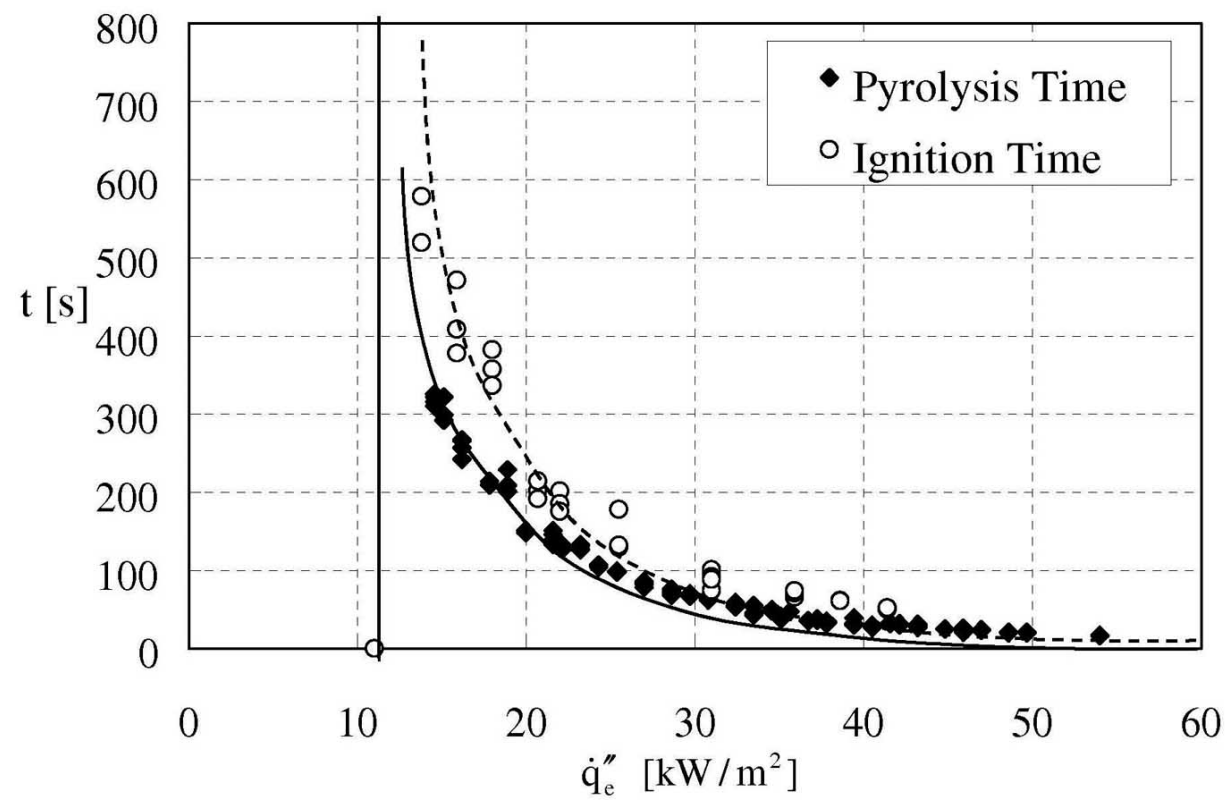

Fig. (2). Ignition ( $\left.t_{\mathrm{ig}}\right)$ and pyrolysis $\left(t_{\mathrm{p}}\right)$ delay times for black PMMA in normal gravity. Tests were conducted using the LIFT (ASTM-1321) and the pyrolysis time was defined as the first observed gases emerging from the surface and visualized by means of a Laser sheet. 
transmitted to solid by conduction. The solid is assumed to be thermally thick and semi-infinite. The initial temperature field and the temperature far from the surface are considered to be equal to the ambient temperature.

The energy balance at the surface of the fuel sample under radiative heating is shown in Fig. (3) and is given by equation (6).

$\dot{q}_{S}^{\prime \prime}(0, t)=a \dot{q}_{e}^{\prime \prime}-\varepsilon \sigma\left(T^{4}(0, t)-T_{\infty}^{4}\right)-h_{c}\left(T(0, t)-T_{\infty}\right)$

Where $\left(\dot{q}_{S}^{\prime \prime}\right)$ is the net heat flux at the surface of the solid fuel sample, (a) is the absorptivity of the solid fuel sample, $(\varepsilon)$ is the emissivity of the solid fuel sample, $(\sigma)$ is the Stefan-Boltzmann constant, $(\mathrm{T}(0, \mathrm{t}))$ is the surface temperature at time $(\mathrm{t}),\left(\mathrm{h}_{\mathrm{c}}\right)$ is the convective heat transfer coefficient, and $\left(\mathrm{T}_{\infty}\right)$ is the ambient temperature.

The classical analysis corresponding to the ignition process assumes a linear approximation for the surface reradiation [6]. The radiative term is then defined as:

$\varepsilon \sigma\left(T^{4}(0, t)-T_{\infty}^{4}\right)=h_{r}\left(T(0, t)-T_{\infty}\right)$

This simplification allows an analytical solution of the one dimensional heat conduction energy equation. Controversy on the effect of this approximation on the analytical determination of the minimum heat flux necessary to attain the pyrolysis temperature is still unresolved. For the experiments conducted in [6], the assumption proved to induce errors lower than $10 \%$ in the determination of the surface temperature of the solid (heat fluxes ranging from 10 to 50 $\mathrm{kW} / \mathrm{m}^{2}$ ). A detailed discussion concerning the influence of this assumption on the determination of the thermal properties of diverse materials can be found in [14]. In short, the author concluded that this assumption leads to over-predict the ignition delay time for low heat fluxes and to overestimate the thermal properties of the materials. However, the results provide a good estimation of the ignition times for high heat fluxes and allow establishing a classification of the ignition properties of materials.

Substituting (7) into (6) and assuming that the total heat transfer coefficient $\left(\mathrm{h}_{\mathrm{T}}\right)$ is equal to the sum of the convective heat transfer coefficient $\left(h_{c}\right)$ and the radiative heat transfer

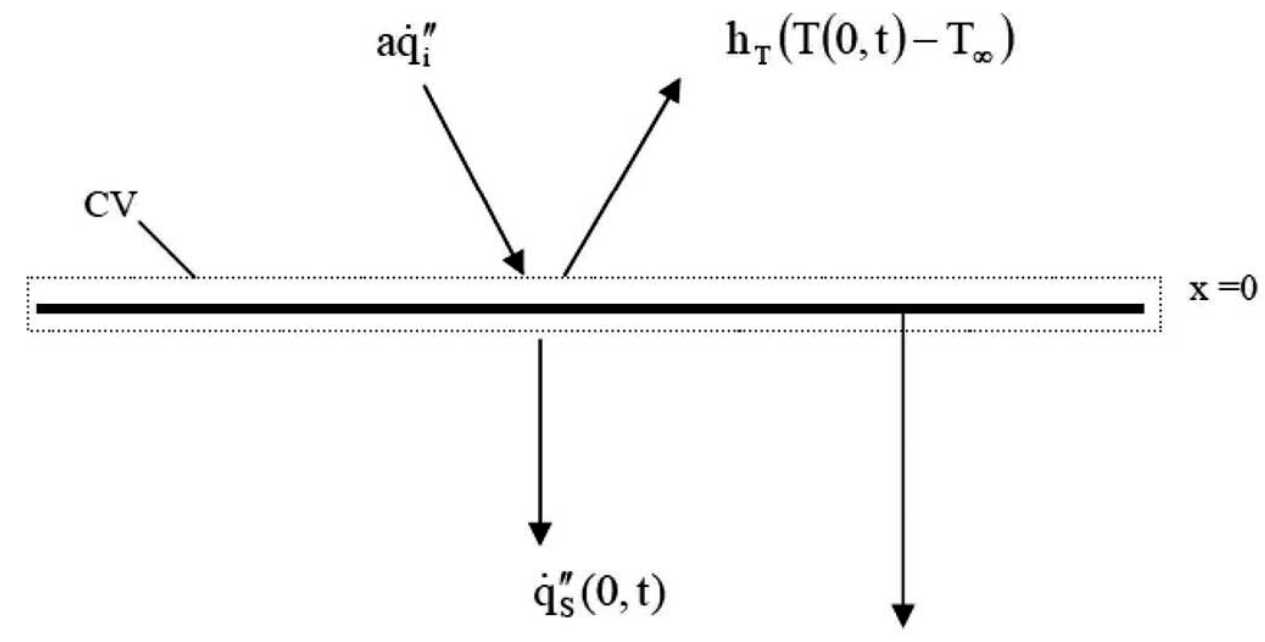

coefficient $\left(\mathrm{h}_{\mathrm{r}}\right)$, the following expression (8) defines the net heat flux $\left(\dot{q}_{S}^{\prime \prime}\right)$ retained near the surface of the solid fuel sample.

$\dot{q}_{S}^{\prime \prime}(0, t)=a \dot{q}_{e}^{\prime \prime}-h_{T}\left(T(0, t)-T_{\infty}\right)$ way

By non-dimensionalizing all variables in the following

$T_{n d}=\frac{T-T_{\infty}}{T_{i g}-T_{\infty}}$

$x_{n d}=\frac{x}{x_{c}}$ where $x_{c}=k / h_{T}$

$t_{n d}=\frac{t}{t_{c}}$ where $t_{c}=k \rho C / h_{T}^{2}$ and

$\dot{q}_{n d}^{\prime \prime}=\frac{\dot{q}^{\prime \prime}}{\dot{q}_{c}^{\prime \prime}}$ where $\dot{q}_{c}^{\prime \prime}=h_{T}\left(T_{i g}-T_{\infty}\right) / a$

the following solution is obtained for the evolution of the temperature in the sample [12]:

$T_{S, n d}=\dot{q}_{e, n d}^{\prime \prime}\left[1-e^{t_{n d}} \operatorname{erfc}\left(\sqrt{t_{n d}}\right)\right]$

To solve for the ignition time $\left(t_{i g, n d}\right)$ a first order Taylor series expansion of equation (9) is conducted. The range of validity of this expansion is limited, thus can not be used over a large range of incident heat fluxes. Consequently, the domain has to be divided at least in two. The first domain corresponds to high incident heat fluxes where the ignition temperature $\left(T_{i g, n d}\right)$ is attained very fast, $t_{i g, n d} \rightarrow 0$. Application of the first order Taylor Series Expansion yields:

$t_{i g, n d}=\frac{\pi}{4} \frac{1}{\left(\dot{q}_{e, n d}^{\prime \prime}\right)^{2}}$

The second domain corresponds to incident heat fluxes close to the critical heat flux for ignition $\left(\dot{q}_{0, i g, n d}^{\prime \prime} \approx 1\right)$ where $\mathrm{x}=0$

Fig. (3). Energy balance at the surface of the solid fuel sample. 


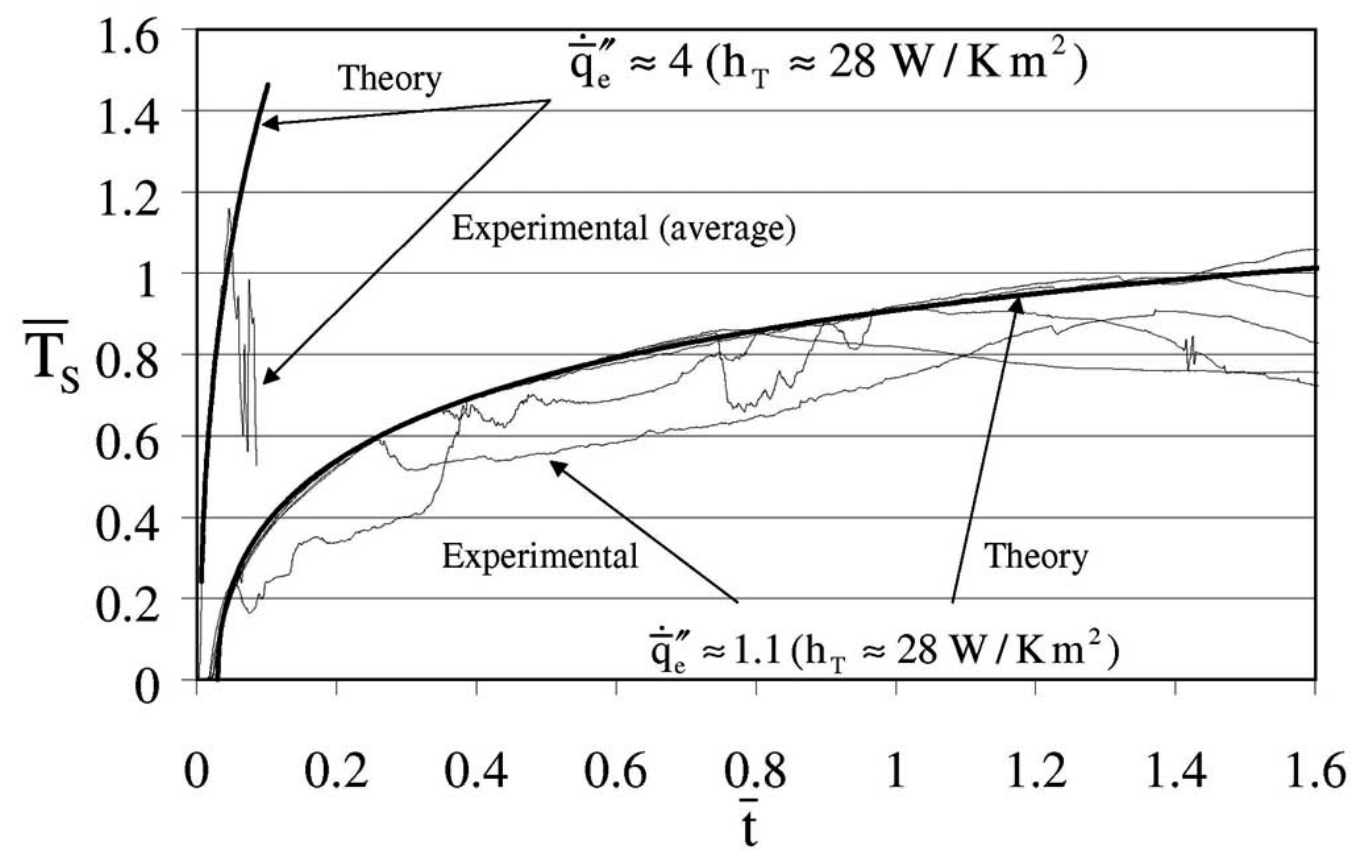

Fig. (4). Evolution of the surface temperature $\left(T_{S, n d}\right)$ with time $\left(t_{n d}\right)$, comparison between the theoretical predictions and the experimental values. For $\dot{q}_{e, n d}^{\prime \prime} \approx 4$, an average experimental value of 32 thermocouple histories (thin line) is compared with the theoretical prediction calculated with $h_{T} \approx 28 \mathrm{~W} / K \mathrm{~m}^{2}$ (thick line). For $\dot{q}_{\boldsymbol{e}, n \widetilde{d}}^{\prime \prime} 1.1$, individual thermocouple histories (thin lines) are compared with the theoretical prediction calculated with $h_{T} \approx 28 \mathrm{~W} / \mathrm{Km}^{2}$ (thick line).

the ignition temperature $\left(T_{i g, n d}\right)$ is attained very slowly, $t_{i g, n d} \rightarrow \infty$.

$t_{i g, n d}=\frac{1}{\pi}\left(\frac{1}{1-1 / \dot{q}_{e, n d}^{\prime \prime}}\right)^{2}$

At $\dot{q}_{0, i g, n d}^{\prime \prime} \approx 1$ the surface will attain the ignition temperature $\left(T_{i g, n d}\right)$ at equilibrium, therefore if $\dot{q}_{e, n d}^{\prime \prime}<\dot{q}_{0, i g, n d}^{\prime \prime} \approx 1$ the surface will never reach the pyrolysis temperature.

The use of a linearized total heat transfer coefficient has been questioned in the literature [9] and corrections that incorporate the non-linear nature of surface re-radiation have been proposed [10]. Temperature histories for different external heat fluxes are presented in Fig. (4). By fitting the theory to the temperature histories a total heat transfer coefficient can be obtained and it can be seen that excellent agreement is found between theory and experiments for a wide range of external heat fluxes ( $\dot{q}_{e, n d}^{\prime \prime} \approx 1.1$ to $\dot{q}_{e, n d}^{\prime \prime} \approx 4$ ). The fitting procedure will be detailed in the following for materials with unknown thermal properties. For $\dot{q}_{e, n d}^{\prime \prime} \approx 4$ and average temperature history is presented but for $\dot{q}_{e, n d}^{\prime \prime} \approx 1.1$ individual recordings are shown.

The individual recordings serve to show the difficulty of acquiring temperature measurements with thermocouples. At a certain point the thermocouples will separate from the surface, this can occur in a random manner (as shown by Fig. 4). While the thermocouple is attached to the surface the temperature follows theory well. The material properties used for PMMA are provided in Table $\mathbf{1}$ and were obtained from different sources listed by Hallman [15] and Steinhaus [16].

For most materials currently used in construction, furnishings and specially those used in aerospace applications, evaluation of the thermal properties of the material is not possible. Therefore the above analysis is fit to experimental evaluation of the ignition delay time (equations (10) and (11)) and the thermal inertia " $\mathrm{k} \rho \mathrm{C}$ " and $\mathrm{T}_{\mathrm{ig}}$ can be evaluated. The fitting procedure consists in: (i) evaluating experimentally the critical flux $\dot{q}_{c}^{\prime \prime}$; (ii) fitting the value of $\mathrm{h}_{\mathrm{T}}$ to obtain a match between the theory and the experimental temperature histories as shown in Fig. (4); (iii) to extract $T_{\text {ig }}$ from the

Table 1. Thermal Properties of Black Poly(Methylmethacrylate) as Compiled by Hallman [15] and Steinhaus [16]. All Properties are Evaluated at an Average Temperature of $373 \mathrm{~K}$

\begin{tabular}{|l|c|}
\hline \multicolumn{1}{|c|}{ Property } \\
\hline \hline $\mathrm{C}[\mathrm{J} / \mathrm{kg} \cdot \mathrm{K}]$ & 2,020 \\
\hline$\rho\left[\mathrm{kg} / \mathrm{m}^{3}\right]$ & 1,180 \\
\hline $\mathrm{k}[\mathrm{W} / \mathrm{m} \cdot \mathrm{K}]$ & 0.192 \\
\hline $\mathrm{a}$ & 0.85 \\
\hline $\mathrm{T}_{\mathrm{ig}}\left[{ }^{\circ} \mathrm{C}\right]$ & 265 \\
\hline $\mathrm{T}_{\infty}\left[{ }^{\circ} \mathrm{C}\right]$ & 20 \\
\hline $\mathrm{h}_{\mathrm{T}}\left[\mathrm{W} / \mathrm{m}^{2} \mathrm{~K}\right]$ & 28 \\
\hline
\end{tabular}




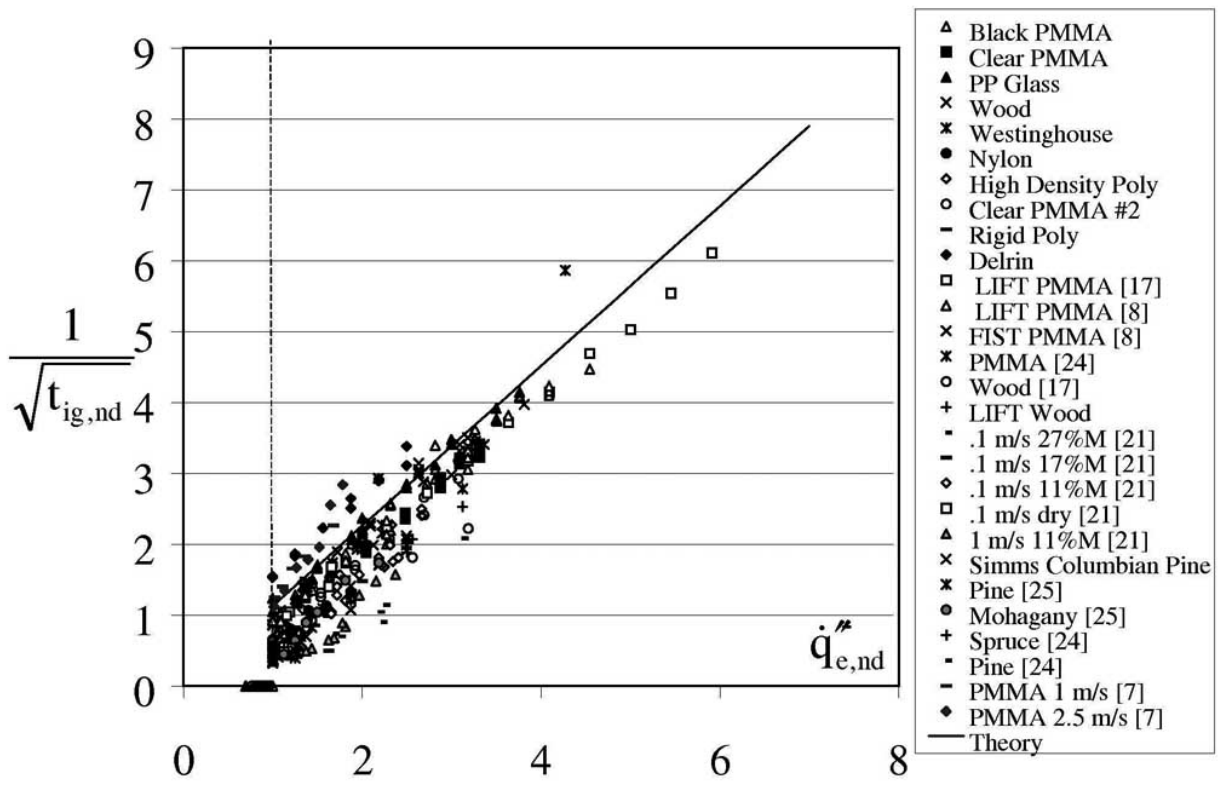

Fig. (5). Evolution of the ignition delay time with the external heat flux. Comparison of experimental data of the present study with the theoretical predictions and data from the literature.

critical heat flux for ignition: $\dot{q}_{c}^{\prime \prime}=\dot{q}_{0, i g, n d}^{\prime \prime}=h_{T}\left(T_{i g}-T_{\infty}\right) / a$; and (iv) to extract the thermal inertia " $\mathrm{k} \rho \mathrm{C}$ " from equations (10) and (11). The emissivity of the material is introduced whenever it can be determined but generally it is assumed to be unity since the materials tend to blacken when exposed to the external heat flux [15]. A series of materials has been tested following conventional protocols $[6,7]$. The data nondimensionalized per equations (10) and (11) is presented in Fig. (5). Table 2 presents the list of materials used and the properties used when correlating the data.

Table 2. Material Properties from Ignition Tests as Obtained from the Ignition Delay Times

\begin{tabular}{|c|c|c|}
\hline Material & $\begin{array}{c}\mathbf{k \rho C} \\
\left(\mathrm{kW} / \mathrm{m}^{2} \mathbf{K}\right)^{2} \mathbf{s}\end{array}$ & $\begin{array}{c}\dot{\bar{q}}_{0, i g}^{\prime \prime} \\
\left(\mathbf{k W} / \mathbf{m}^{2}\right)\end{array}$ \\
\hline LIFT Wood [7] & 0.29 & 16 \\
\hline LIFT Wood & 0.17 & 16 \\
\hline FIST Wood [12] & 0.14 & 16 \\
\hline LIFT black PMMA [7] & 2.08 & 9 \\
\hline LIFT black PMMA & 1.40 & 11 \\
\hline FIST black PMMA [12] & 1.24 & 11 \\
\hline Clear PMMA & 0.58 & 12.5 \\
\hline Delrin & 0.59 & 16 \\
\hline High Density Polyethylene & 0.46 & 15 \\
\hline Nylon & 0.13 & 25 \\
\hline Rigid Polyethylene & 0.12 & 24 \\
\hline PP/Glass Composite & 0.91 & 10 \\
\hline Clear PMMA \#2 & 0.56 & 13 \\
\hline Westinghouse Glass/Epoxy Laminate & 0.52 & 18 \\
\hline
\end{tabular}

The previous data show that the model can be applied to a large range of materials. More materials can be found in [6-13]. However, due to the limitations in the model presented here above, the applicability of the methodology has to be tested for each new considered material. This is the case for the forest fuel presented here after.

\section{Considerations Pertaining to Scaling}

When ignition is conducted under conditions that are not typical of the standard test [6,7] convective heat and mass transfer is modified due to the characteristics of the environment and the length scale of the heated sample. Equation (4) might no longer be simplified to $t_{i g} \approx t_{p}$ and the effect of a variation of convective transport needs to be evaluated. The convective heat transfer coefficient can vary from approximately $20 \mathrm{~kW} / \mathrm{m}^{2} \mathrm{~K}$ to $2 \mathrm{~kW} / \mathrm{m}^{2} \mathrm{~K}$ [12] leading to a reduction in $\mathrm{t}_{\mathrm{p}}$ and $\dot{q}_{c}^{\prime \prime}$. The reduction of $\mathrm{h}_{\mathrm{T}}$ will have a decreasing effect on the total ignition delay time as the external heat flux increases and can be incorporated in the theoretical development that leads to equation (10). A perfect example of an environment where the reduction in convective motion can result in the breakdown of these assumptions is microgravity. Experimental results reported by Roslon et al. [17] show that for PMMA and a polypropylene/glass composite the ignition delay time decreases significantly (up to $50 \%$ in the some cases). The significant variation of the ignition delay time can be attributed to the combined effect of varying the time to attain pyrolysis and the mixing time. Under the assumption that ignition will occur when a flammable mixture is attained (lean flammability limit), a reduction in convective transport implies a reduction in $t_{m}$. Therefore the mixing time $\left(\mathrm{t}_{\mathrm{m}}\right)$ needs to be analyzed and, in the presence of a strong pilot, equation (1) can be only reduced to $t_{i g} \approx t_{p}+t_{m}$. 
It was shown by Long et al. [12] that, under normal gravity conditions, the fuel mass fraction can be obtained by means of an integral analysis of the boundary layer formed upstream of the pilot. Thus the fuel mass fraction at the pilot $\left(\mathrm{Y}_{\mathrm{F}}\right)$ can be defined as:

$$
Y_{F}=\frac{\dot{m}_{F}^{\prime}}{\dot{m}_{F}^{\prime}+\dot{m}_{O}^{\prime}}
$$

Where $\dot{m}_{F}^{\prime}$ and $\dot{m}_{O}^{\prime}$ are the mass flux of fuel and oxidizer respectively, integrated over the stream wise coordinate. Long et al. [12] proposed a model to determine $\mathrm{Y}_{\mathrm{F}}$ and showed that ignition occurred at a constant value of the fuel mass fraction that they labelled the lean flammability limit, $\mathrm{Y}_{\mathrm{FL}}$. This interpretation could serve to predict the ignition delay time in micro-gravity but the uncertainty in the flow structure during the parabolic flight experiments reported by Roslon et al. [17] make this comparison difficult. Long term micro-gravity experiments will allow a better validation of theory with experimental results.

\section{AN APPLICATION TO FOREST FIRES}

Forest fires are the typical example of the difficulty of applying scaling and defining laws correctly. Equations (2) and (3) have been tested for laboratory experiments [18] but it is difficult to extend the results at the actual scale of forest fires. The flame characteristics, as well as the temperature distribution in the fire plume have proved to differ between the field and the lab [19], due to the different length scales for vegetation and turbulence.

Vegetation is very different from the usual fuels encountered in human-developed environments. It is an extremely porous fuel $(2-5 \%$ of the volume is occupied by the solid phase in pine needle litters, $2-5 \%$ in shrub canopies and $0.2-$ $0.5 \%$ in tree canopies) $[20,21]$. This live fuel is also in equilibrium with the ambient and it is changing very quickly with the external conditions. The most obvious change is moisture content [22] but Volatile Organic Compound production is also changing in function of soil, weather conditions and species [23]. However, the previous analysis for fuel ignition has been applied to pine needle beds as they are the most common forest floor fuel in pine forest, which are widely spread not only in the Mediterranean but also in NorthAmerica and Russia.

Ignition of forest fuels is critical to study wildfire behavior. A classification of ignition times as a function of species and parameters such as moisture content can lead to the development of improved fire risk assessment tools [24]. The theory developed in this paper has been developed mainly for solid fuels and its extension to forest fires is very interesting. However, the range of validity of the theory has to be assessed for vegetable fuels and some hypotheses of the model seem to be a priori challenged: The fuel matrix is a multiphase medium mainly constituted by air; the radiative heat insult is not only present at the surface but is going through the porous fuel layer; the surface and inner temperatures are not unique in the multiphase medium; convective transfers can develop inside the fuel layer. However, the aim of the study is to evaluate if these new conditions change dramatically the basic premises of the model or if the model is still valid in a given range of conditions.

\section{Experimental Protocol}

The experiments presented in this paper were conducted using the FM-Global Fire Propagation Apparatus (FPA) [25]. For this study, radiative heat fluxes imposed to the sample varied between $8-50 \mathrm{~kW} \cdot \mathrm{m}^{-2}$. The FPA allows natural convection or forced gas flow rate through the fuel bed. Specific sample holders were used for the experiments [26]. Indeed, dead or living vegetable fuels are very porous and flow can develop inside the fuel layer, acting strongly on the combustion dynamics. They consisted in circular baskets, made of stainless steel, with holes on all the surfaces (sides and bottom), to allow flow to pass through the bed of pine needles. Three different percentage openings have been test: $0 \%$ (one basket lined with aluminium foil), $26 \%$, and $63 \%$. The studied fuel was dead and not conditioned Pinus halepensis $(\mathrm{Ph})$ needles. The moisture levels have been determined by oven drying of samples for 24 hours at $60^{\circ} \mathrm{C}$. The percentage of humidity of the fuel samples ranged between $4.9 \%$ and $6.4 \%$. The baskets were filled to the top and had a constant mass of $15 \mathrm{~g}$, inducing a fuel bed porosity of $95 \%$. The experimental results that have been used are from [26-28], plus some original to this study.

\section{Results}

Fig. (6) displays the ignition times for different heat insults. The curve in Fig. (6) is steeper than the one in Fig. (2), due to the thermal characteristics of the fuel. Indeed, particles are thermally thin and they heat up very quickly when submitted to a heat insult.

However, the results are consistent with Fig. (2), particularly for the $0 \%$ baskets. Closed baskets, while preventing a convective flow to establish in the fuel bed, provide results similar to solid fuels. The results obtained with $26 \%$ and $63 \%$ baskets show the same tendency but they are more scattered as natural convection is allowed inside the sample [26]. As the heat insult increases, the scattering becomes less important and the ignition time becomes very short. In this case, the results are more difficult to analyze because the times are very short and there is a competition between two effects due the porosity of the samples. Firstly, the degradation gases are diluted by the air coming from the bottom of the baskets. This effect tends to increase the ignition time. Secondly, the degradation gases mix with air inside the sample. This effect tends to provide a flammable mixture next to the pilot flame earlier in the process, which would result in decreasing the ignition time.

The main difference between Figs. (2) and (6) is to be found in pyrolysis times. They are much lower for pine needles than for solid fuels. Smoke emission was also observed for $\dot{q}_{e}^{\prime \prime}=8 \mathrm{~kW} / \mathrm{m}^{2}$, with no ignition (the critical flux has found to be $\dot{q}_{c}^{\prime \prime}=8.5 \mathrm{~kW} / \mathrm{m}^{2}$ ). This behavior can be explained by different factors: the fuel contains some water (4.9-6.4\% of moisture content for the whole range of experiments) that is released at low temperatures; the needles are thermally thin and heat up very quickly at the top of the fuel bed, pyrolysing very quickly but not releasing enough flammable gases to allow ignition. Given that, no flash ignition was observed for $\dot{q}_{e}^{\prime \prime}=8 \mathrm{~kW} / \mathrm{m}^{2}$ and it can be concluded that not enough fuel was being produced to enter the 


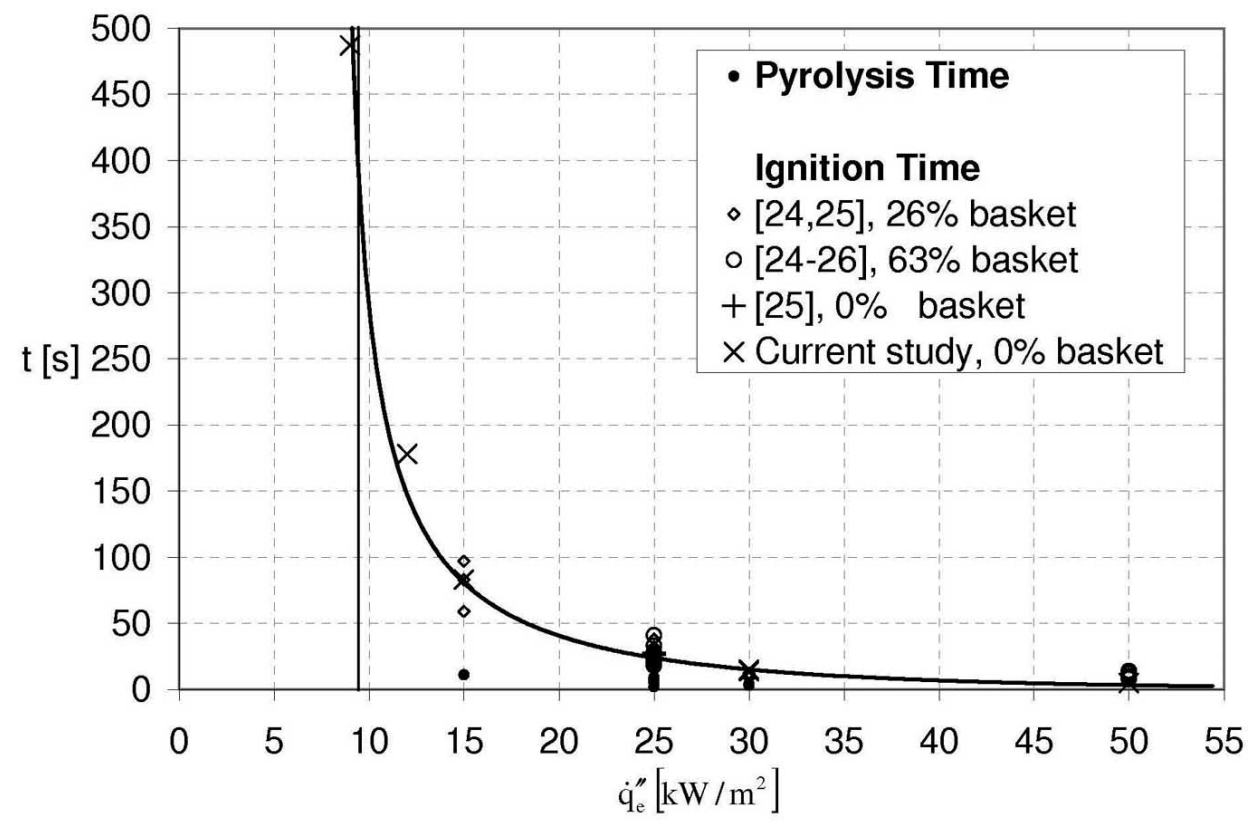

Fig. (6). Ignition $\left(t_{i g}\right)$ and pyrolysis $\left(t_{p}\right)$ delay times for pine needles. The pyrolysis time was defined as the first gases visually observed emerging from the surface.

flammability range. This phenomenon has already been discussed in the previous sections but for pine needles, the effect seems to be more important. Indeed, it is enhanced by the high porosity of the sample (95\%) and its low mass, both contributors to dilution. However, ignition and smoke production times for higher heat flux values, that correspond to the usual conditions of fire spread [29,30], are very short and very close (see $30 \mathrm{~kW} / \mathrm{m}^{2}$ in Fig. 6) and they almost overlap.

Fig. (7) displays the evolution of the surface temperature of the sample for a heat flux close to the critical one. The parameters of the model are obtained from the $0 \%$ basket data as follows:

- $t_{i g}$ is obtained thanks to equation (10).

- $\mathrm{h}_{\mathrm{T}}$ is set by fitting the solution to the experimental temperature curves as described for solid fuels in the previous section.

- $\mathrm{k} \rho \mathrm{C}$ and $\mathrm{T}_{\mathrm{ig}}$ are calculated thanks to equation (11) and $\dot{q}_{c}^{\prime \prime}=\dot{q}_{0, i g, n d}^{\prime \prime}=h_{T}\left(T_{i g}-T_{\infty}\right) / a$, respectively.

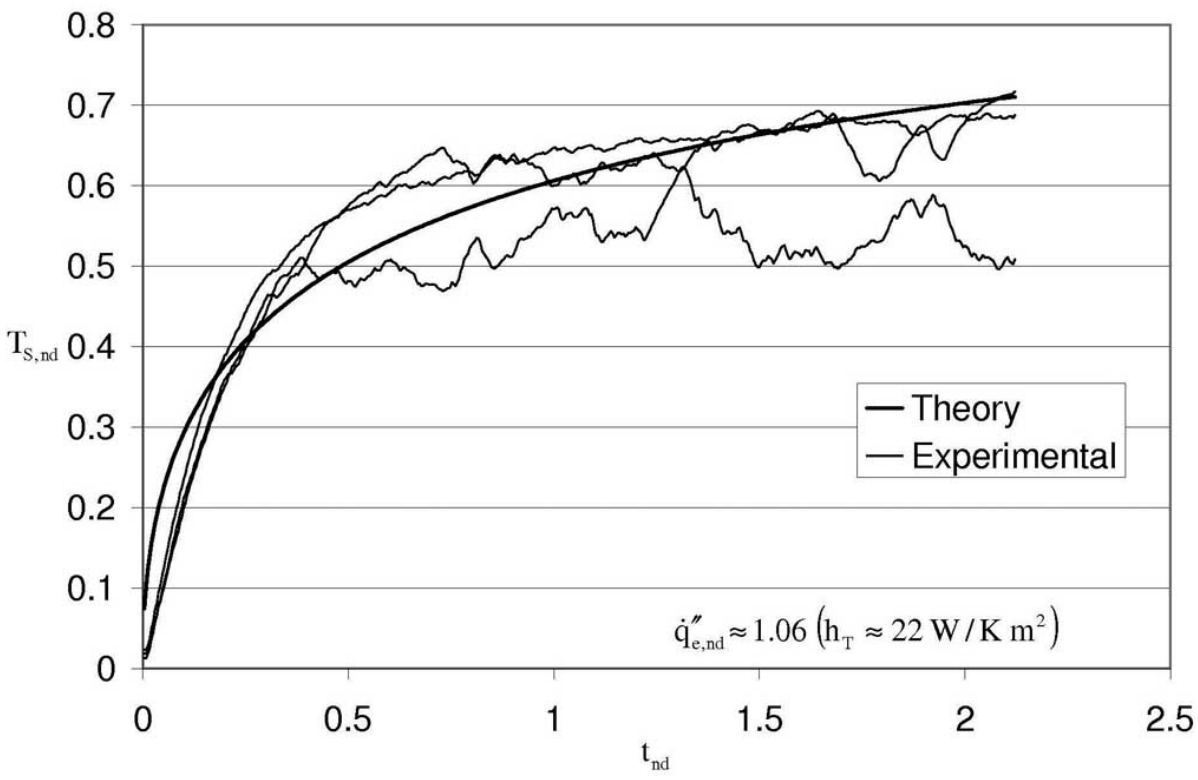

Fig. (7). Evolution of the "surface" temperature $\left(T_{S, n d}\right)$ with time $\left(t_{n d}\right)$, comparison between the theoretical predictions and the experimental values for $\dot{q}_{e, n d}^{\prime \prime}=1.06$, individual thermocouple histories (thin lines) are compared with the theoretical prediction calculated with $h_{T} \approx 22 \mathrm{~W} / \mathrm{Km}^{2}$ (thick line). 
The theoretical curve provides less agreement with experimental curves than in Fig. (2) as the surface temperature of the pine needle bed is not well defined. Indeed, in the case of Fig. (3), the thermocouples are touching the surface of the PMMA sample while for Fig. (7) the thermocouples are at the top of a fuel bed, in contact with both pine needles and air.

The increase of temperature at the beginning of the test is not relevant as it is more representative of the thermocouples than of the fuel bed. Indeed, the thermal inertia of the thermocouples is higher than the one of the pine needles.

However, for a long time (ignition occurred at $487 \mathrm{~s}$ ), the thermocouple is close to being in thermal equilibrium with the fuel bed and the temperature is more relevant. For high values of $\dot{q}_{e, n d}^{\prime \prime}$, the discrepancy is even bigger as ignition is occurring very quickly and the thermocouples are not able to track the steep increase of temperature of the fuel.

Table 3 displays the results obtained from the procedure described here below. The ignition temperature found in literature for pine needles is between $280-350^{\circ} \mathrm{C}$ [31]. An estimation of " $\mathrm{k} \rho \mathrm{C}$ " thanks to literature [31,32] provides a value of $0.09\left(\mathrm{~kW} / \mathrm{m}^{2} \mathrm{~K}\right)^{2} \mathrm{~s}$. These values are very different from the values obtained in Table 3, particularly for "k $\rho \mathrm{C}$ ", even if one considers a correction factor to estimate the actual values from the effective ones, as done by Mowrer [14]. It should be noticed that the value of the parameters do not represent the actual physical values but the value of the control parameters of the system [33]. However, the estimated parameters should be in the range of the physical parameters. It is the case for $\mathrm{T}_{\mathrm{ig}}$ but, for " $\mathrm{k} \rho \mathrm{C}$ ", the first estimation has been done only considering the equivalent conductivity of the fuel bed that is very low because it is constituted by $95 \%$ of air. An estimation of the radiative transfer through the fuel bed has been done by linearizing it inside the porous medium [34] and the estimated " $\mathrm{k} \rho \mathrm{C}$ " is equal to $0.105\left(\mathrm{~kW} / \mathrm{m}^{2} \mathrm{~K}\right)^{2} \mathrm{~s}$, which is a more realistic value.

Fig. (8) presents the evolution of the ignition delay time with the external heat flux. The closed baskets $(0 \%)$ display a good agreement with the theory. This result is almost unexpected as the assumptions of the model were strongly challenged (multiphase medium, surface temperature, conductive transfer through the fuel bed and radiative boundary condition). However, it seems that the prevention of convective transfer in the fuel samples allows matching the theory. The results are even better than many others found with solid fuels. This means that even if the particles are thermally thin, the bulk properties of the sample induce a behavior equivalent to solid fuels. Indeed, the samples used for this paper are representative of forest floors and the radiative transfer through the porous fuel bed behaves like the conductive transfer through a solid.

Open baskets $(26 \%$ and $63 \%)$ show different behaviors. Under natural convection, the samples submitted to normalized heat flux up to the value of 4 follow roughly the theory with more scattering. The reasons of this scattering have already been discussed for Fig. (6). For high fluxes and 63\% baskets, the theory does not match the experiments. The experimental results display a change in regime that can be attributed to an increase in convective transfers. Indeed, the surface of the samples is heated very quickly and buoyancy is enhanced. To highlight this change in regime, experimen-

Table 3. Estimated Material Properties from Ignition Tests as Obtained from the Ignition Delay Times

\begin{tabular}{|c|c|c|c|c|}
\hline Material & $\begin{array}{c}\mathbf{k} \rho \mathbf{C} \\
{\left[\left(\mathbf{k} / \mathbf{m}^{2} \mathbf{K}\right)^{2} \mathbf{s}\right]}\end{array}$ & $\begin{array}{c}\mathbf{h}_{\mathrm{T}} \\
{\left[\mathbf{W} / \mathbf{m}^{2} \mathbf{K}\right]}\end{array}$ & $\begin{array}{c}\mathbf{T}_{\mathrm{g}} \\
{\left[{ }^{\circ} \mathbf{C}\right]}\end{array}$ & $\begin{array}{c}\mathbf{t}_{\mathbf{c}} \\
{[\mathbf{s}]}\end{array}$ \\
\hline \hline Ph needle bed & 0.11 & 22 & 412 & 230 \\
{$\left[\mathbf{k W} / \mathbf{m}^{2}\right]$}
\end{tabular}

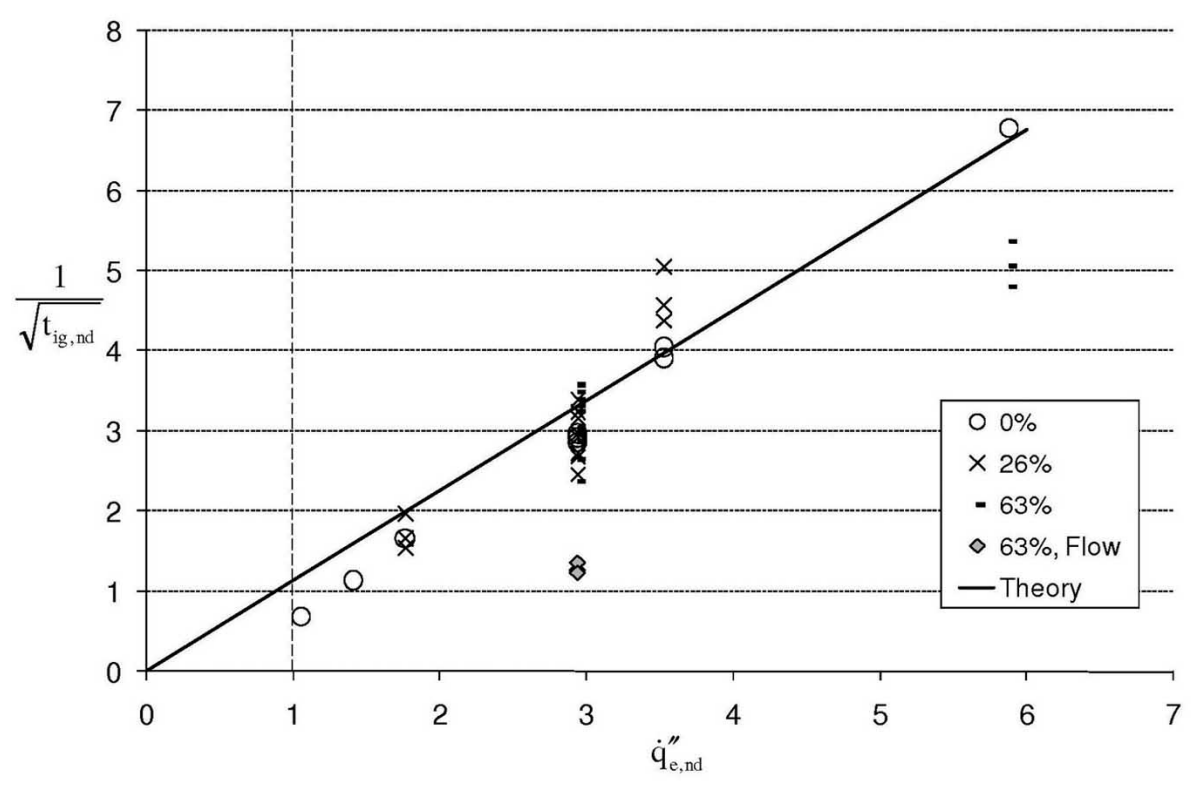

Fig. (8). Evolution of the ignition delay time with the external heat flux. Comparison of experimental data with the theoretical predictions ([26-28] and present study). 
tal results for a forced flow and a $63 \%$ basket (normalized heat flux around the value of 3) has been added to the Figure. The times to ignition are much longer than the ones predicted by the theory as convection is dramatically increased.

The dilution of the flammable gases is also increased. This effect has been visually observed thanks to the behavior of the smoke above the fuel bed before ignition. It was clearly dragged away from the top of the fuel bed by the flow.

\section{Considerations Pertaining to Scaling}

The comments given in the previous section are still valid in the case of forest fuels. However, for the highly porous vegetable fuels, flow and convective transfers inside the fuel layer are supplementary effects that can have a strong influence on ignition times.

In the case of forest floors, the scale of the phenomenon is not much bigger than the one of the experiments used in this study. As long as convective transfers are low, one can expect to obtain equivalent results (this could be the case near the ground under dense forests).

For a dense and low shrub layer, it could be also the case. However, the porosity is one order of magnitude higher than for litters and the assumption of a linearized radiation transfer through the layer has to be confirmed.

For high convective transfer, the mixture time $t_{m}$ has to be estimated in accordance with the flow. Furthermore, vegetable live fuels have high moisture contents and a supplementary induction time, due to the dehydration of the fuel, has to be included. This process in endothermic and plays also a role on the thermal balance (5).

In the case of crown fires, the problem is even more difficult as the porosity is two orders of magnitude higher than in litters. A linearized radiation is most likely a poor assumption [35].

All these effects are less studied in literature than for usual fire safety problems and dedicated studies have to be developed for each configuration.

\section{CONCLUSIONS}

The process of ignition has been used to define the importance of different scaling parameters. The scaling parameters put in evidence the different material properties and non-dimensional groups controlling these processes. Their limitations have been assessed in the context of different environmental conditions and available experimental data. It has been shown that for a broad range of experimental conditions these parameters provide a robust description of the driving mechanisms, which can be useful for fuel classification and for the estimation of ignition times for fuels submitted to high heat fluxes.

The extension to forest fires provides the basis of the study of risk indexes for fire ignition and fire spread. The theory provides good results as long as the influence of the flow inside the fuel layer is negligible on both convective transfer and pyrolysis gases dilution. In this case, equation (5) remains valid but the thermal transfer through the fuel bed is mainly due to radiation and not to conduction. A further step in the study of forest fuels, as well as of highly po- rous materials, would be to characterize the change in regime from diffusive (conductive and/or radiative) to convective transfer and to develop a model which would be able to take into account this change.

\section{ACKNOWLEDGEMENTS}

The work related to the LIFT was funded by NASA Glenn under grant NAG-31961. The authors wish to thank Prof. A.C. Fernandez-Pello and co-workers at the University of California, Bekeley for years of discussion. JLT was funded by FM Global through student support and the donation of the FPA The contributions of the many graduate students involved in these projects have made them possible. Nicolas Bal and Hubert Biteau, both PhD students at the University of Edinburgh, helped to conduct the original experiments for this paper.

\section{REFERENCES}

[1] J.G. Quintiere, "Scaling applications in fire research," Fire Safety Journal, 15, pp.3-29, 1989.

[2] E.E. Zukoski, "Properties of fire plumes," Chapter 3, Combustion fundamentals of Fire, G. Cox Editor, Academic Press, pp.101-220, 1995.

[3] P. Joulain, "Behavior of pool fires: state of the art and new insights," Combustion Institute, Symposium (International) on Combustion, 27th. Proceedings., vol. 2. August 2-7, 1998, Boulder, CO, Combustion Institute, Pittsburgh, PA, 2691-2706 pp, 1998.

[4] SFPE Handbook of Fire Protection Engineering, $3^{\text {rd }}$ ed. Society of Fire Protection Engineers, Philip J.N, Ed. National Fire Protection Association, Quincy, Massachusetts, USA 2002.

[5] D.D. Drysdale, "An Introduction to fire dynamics," $2^{\text {nd }}$ ed. Wiley, New York, 1999.

[6] J.G. Quintiere, "A simplified theory for generalizing results from a radiant panel rate of flame spread apparattus," Fire and Materials, vol. 5, no.2, pp. 52-60, 1981.

[7] J. G. Quintiere, Harkleroad, M., "New concepts for measuring flame spread properties," NBSIR-84-2943, National Bureau of Standards, 1984.

[8] I. S. Wickman, "Theory of opposed-flow flame spread," Progress in Energy and Combustion Science, vol. 18, issue, 6, pp. 553-593, 1992.

[9] Janssens, M. L., "Thermal model for piloted ignition of wood including variable thermophysical properties," Fire Safety Science$3^{\text {rd }}$ International Symposium, 167-176, 1991.

[10] T. Kashiwagi, "Effects of sample orientation on radiative ignition," Combustion and Flame, vol. 44, pp. 223-245, 1982.

[11] A. Atreya, C. Carpenter, M. Harkleroad, "The effect of sample orientation on piloted ignition and flame spread," Fire Safety Science - $1^{\text {st }}$ International Symposium, pp.97-109, 1985.

[12] R.T Long, J.L. Torero, J.G. Quintiere, A.C. Fernandez-Pello, "Scale and transport considerations on piloted ignition of pmma," Sixth International Symposium on Fire Safety Science, 1999, Poitiers, France.

[13] J.L. Cordova, D. C. Walther, J. L. Torero, A.C. Fernandez-Pello, "Oxidizer flow effects on the flammability of solid combustibles," Combustion Science and Technology, vol. 164, no. 1-6, pp. 253278, 2001.

[14] F.W. Mowrer, "An analysis of effective thermal properties of thermally thick materials", Fire Safety Journal, vol. 40, pp.395-410. 2005 .

[15] J. Hallman, "Ignition characteristics of plastics and rubber," $\mathrm{Ph} . \mathrm{D}$ Thesis, University of Oklahoma, Norman, OK, USA, 1971.

[16] T. Steinhaus, "Evaluation of the thermophysical propertiesof poly(methylmethacrylate): a reference material for the development of a flammability test for micro-gravity environments," M.S. Thesis, University of Maryland, 1999.

[17] M. Roslon, S. Olenick, D. Walther, J.L.Torero, A.C. FernandezPello, H. D. Ross, "Microgravity ignition delay of solid fuels in low velocity flow," AIAA-2000-0580, 2000.

[18] F. Morandini, A. Simeoni, P.A. Santoni, J.H. Balbi, "A model for the spread of fire across a fuel bed incorporating the effects of wind 
and slope", Combustion Science and Technology, vol. 177, pp. 1381-1418, 2005.

[19] P.A. Santoni, A. Simeoni, J.L. Rossi, F. Bosseur, F. Morandini, X. Silvani, J.H. Balbi, D. Cancellieri, L. Rossi, "Instrumentation of wildland fire: characterisation of a fire spreading through a Mediterranean shrub", Fire Safety Journal, vol. 41, no. 3, pp. 171-184, 2006.

[20] P.M. Fernandes, E. Rigolot, "The fire ecology and management of maritime pine (Pinus pinaster Ait.)", Forest Ecology and Management, vol. 241, pp. 1-13, 2007.

[21] J.M.C. Pereira, N.M.S. Sequeira, J.M.B. Carreiras, "Structural properties and dimensional relations of some mediterranean shrub fuels", International Journal of Wildland Fire, vol. 5, no. 1, pp. 3542, 1995.

[22] E.A. Catchpole, W.R. Catchpole, N.R. Viney, W.L. McCaw, J.B. Marsden-Smedley, "Estimating fuel response time and predicting fuel moisture content from field data", International Journal of Wildland Fire, vol. 10, pp. 215-222, 2001.

[23] A.P. Dimitrakopoulos, P.I. Panov, "Pyric properties of some dominant Mediterranean vegetation species", International Journal of Wildland Fire, vol. 10, pp. 23-27, 2001.

[24] E. Ormeno, B. Cespedes, I.A. Sanchez, A. Velasco-Garc1, J.M Moreno, C. Fernandez, V. Baldy, "The relationship between terpenes and flammability of leaf litter", Forest Ecology and Management, vol. 257, pp. 471-482, 2009.

[25] "Standard test method for measurement of synthetic material flammability using a fire propagation apparatus", ASTM, E205803, 2003.

[26] C. Schemel, A. Simeoni, H. Biteau, J. Riviera, J.L. Torero, "A calorimetric study of wildland fuels", Experimental Thermal and Fluid Science, vol. 32, n0. 7, pp. 1381-1389, 2008.

[27] P. Bartoli, A. Simeoni, H. Biteau, J.L. Torero, P.A. Santoni, "Determination of the main parameters influencing forest fuel combus- tion dynamics", Sixth Mediterranean Combustion Symposium, 2009, Porticcio, France.

[28] F.X. Jervis, G. Rein, A. Simeoni, J.L. Torero, "The role of moisture in the burning of live and dead pine needles", Interflam, 2010, 5-7 July 2010, Nottingham, UK.

[29] J.D. Cohen, "Relating flame radiation to home ignition using modeling and experimental crown fires", Canadian Journal of Forest Research, vol. 34, pp. 1616-1626, 2004.

[30] X. Silvani, F. Morandini, "Fire spread experiments in the field: Temperature and heat fluxes measurements", Fire Safety Journal, vol. 44, n0. 2, pp. 279-285, 2009.

[31] "Deliverable D-02-02 A: Physical, chemical and thermal characteristics of the wildland fuel particles - Answer from the partners", EUFIRELAB: A Wall-Less Laboratory for Wildland Fire Sciences and Technologies in the Euro-Mediterranean Region, EU FP5 Program EVR1-CT-2002-40028, 2004.

[32] V. Tihay, A. Simeoni, P.A. Santoni, L. Rossi, J.P. Garo, J.P. Vantelon, "Experimental study of laminar flames obtained by the homogenization of three forest fuels" International Journal of Thermal Sciences, vol. 48, n0. 3, pp. 488-501, 2009.

[33] J.L. Torero, "Ignition of solids", in SFPE Handbook of Fire Protection Engineering, $4^{\text {th }}$ ed. Society of Fire Protection Engineers. Philip JD, Ed. National Fire Protection Association, Quincy, Massachusetts, USA, 2008.

[34] P.A. Santoni, "Outils Théoriques, Numériques et Expérimentaux pour l'Etude des Feux de Forêt", Habilitation à Diriger les Recherches Thesis, University of Corsica, 2003.

[35] M.G. Cruz, B.W. Butler, M.E. Alexander, J.M. Forthofer, R.H. Wakimoto, "Predicting the ignition of crown fuels above a spreading surface fire. Part I: model idealization", International Journal of Wildland Fire, vol. 15 no. 1, pp. 47-60, 2006.

(C) Torero and Simeoni; Licensee Bentham Open.

This is an open access article licensed under the terms of the Creative Commons Attribution Non-Commercial License http://creativecommons.org/licenses/by$\mathrm{nc} / 3.0 /$ ) which permits unrestricted, non-commercial use, distribution and reproduction in any medium, provided the work is properly cited. 